\section{Neuroma mentoniano traumático. Reporte de caso clínico}

\section{Traumatic mental neuroma. Clinical case report}

\section{Resumen}

El neuroma traumático no es una verdadera neoplasia sino una proliferación reactiva del tejido neural después de la transección u otro daño de un haz de nervios. Pueden desarrollarse en cualquier lugar, pero son más comunes en el área del foramen mentoniano, la lengua y el labio inferior. La formación de neuromas traumáticos es una causa importante de dolor neuropático, que sigue siendo un problema difícil al que se enfrentan los cirujanos. El mecanismo exacto del dolor asociado al neuroma aún no se comprende completamente y la prevención de su formación es primordial para evitarlo. Hasta la fecha el tratamiento más efectivo ha sido la extirpación quirúrgica. Se reporta el caso de un paciente de sexo femenino de 23 años de edad que presenta un aumento de volumen en el fondo de surco vestibular a nivel de los dientes 44 y 45, así como dolor espontáneo de tipo ardoroso con una evolución de aproximadamente cinco meses. Se decide realizar la resección quirúrgica de la lesión y el resultado histopatológico fue de un neuroma traumático. A los 7 días de seguimiento, se observa un ligero edema en la región bucal y mentoniana; así como anestesia a nivel de la piel del mentón y labio inferior. A 10 meses de seguimiento persistió la anestesia y no se observaron datos de recidiva.

Palabras clave: Neuroma; Trauma; Labio (fuente: DeCS BIREME).

\section{Caso Clínico}

Oskar Eduardo Prada Vidarte ${ }^{1, b}$, Alejandro Alonso Moctezuma ${ }^{1, c}$, José de Jesús Ramos Nieto ${ }^{2, a}$, Roberto Onner Cruz Tapia ${ }^{2, c}$

${ }^{1}$ Universidad Nacional Autónoma de México, Facultad de Odontología, Departamento de Cirugía Oral y Maxilofacial, División de Estudios de Posgrado e Investigación, Ciudad de México, México.

2 Universidad Nacional Autónoma de México, Facultad de Odontología, Departamento de Patología Bucal, División de Estudios de Posgrado e Investigación, Ciudad de México, México.

a Cirujano Dentista.

${ }^{\text {b } M a e s t r i ́ a ~ e n ~ E s t o m a t o l o g i ́ a . ~}$

c Maestría en Ciencias.

\section{Correspondencia:}

Alejandro Alonso Moctezuma: cmfalonsomoctezuma@ hotmail.com

Circuito de los Institutos s/n Ciudad Universitaria, Col. Copilco, Alcaldía Coyoacán, 04510

Código postal: 04510

ORCID: 0000-0001-6265-6636

\section{Coautor:}

Oskar Eduardo Prada Vidarte: eduline22@hotmail.com ORCID: 0000-0002-8827-6209

José de Jesús Ramos Nieto: venison.13@hotmail.com ORCID: 0000-0002-9599-6538

Roberto Onner Cruz Tapia: rob-onner@hotmail.com ORCID: 0000-0002-0633-0436

\section{Editora:}

Sandra Patricia Palomino-Gómez

Universidad Nacional Mayor de San Marcos, Perú.

Conflicto de intereses: los autores declaran no tener conflictos de interés.

Fuente de financiamiento: autofinanciado.

\section{Recibido: $11 / 03 / 20$}

Aceptado: $23 / 07 / 20$

Publicado: 16/11/20

\begin{abstract}
Traumatic neuroma is not a true neoplasm but rather a reactive proliferation of neural tissue after transection or other damage to a nerve bundle. They can develop anywhere but are most common in the area of the mental foramen, tongue, and lower lip. Traumatic neuroma formation is a major cause of neuropathic pain, which remains a difficult problem for surgeons. The exact mechanism of the pain associated with the neuroma is still not fully understood and the prevention of its formation is essential to avoid it. To date the most effective treatment has been surgical removal. We report the case of a 23-year-old female patient who presented an increase in the volume of the vestibular sulcus at the level of teeth 44 and 45, as well as spontaneous burning pain with an evolution of approximately five months. It was decided to perform the surgical resection of the lesion and the histopathological result was a traumatic neuroma. At 7 days of follow-up,
\end{abstract}


slight edema is observed in the oral and mental region; as well as anesthesia at the level of the skin of the chin and lower lip. A 10-month follow-up anesthesia persisted, and no recurrence data were observed.

Keywords: Neuroma; Trauma; Lip (source: MeSH NLM).

\section{Introducción}

El neuroma traumático (NT) no es una verdadera neoplasia sino una proliferación reactiva del tejido neural después de la transección u otro daño de un haz de nervios ${ }^{1,2}$. Puede ser provocado por una historia de trauma o después de la extracción dental u otros procedimientos quirúrgicos. Si estos elementos regeneradores encuentran tejido cicatricial o no pueden restablecer la inervación, entonces puede desarrollarse una masa tumoral en el lugar de la lesión ${ }^{1}$. Dentro de las patologías que se asemejan a los neuromas traumáticos (NTs), encontramos al neuroma mucoso, neurofibroma, neuroma en empalizada y el hamartoma neurovascular ${ }^{3}$.

Los NTs intraóseos pueden mostrar un defecto radiotransparente en las radiografías orales. Estos se presentan en una cuarta parte de los casos y su localización más frecuente es en la región posterior mandibular, a comparación de los NTs extraóseos que se originan en el área del foramen mentoniano, el labio inferior y la lengua ${ }^{1,4}$. Pueden aparecer NTs en otros sitios de cabeza y cuello; se ha estimado que los NTs del nervio auricular mayor se desarrollan en el $5 \%$ al $10 \%$ de los pacientes sometidos a cirugía para los adenomas pleomórficos de la glándula parótida ${ }^{1}$.

Los NTs pueden aparecer a cualquier edad, pero se diagnostican con mayor frecuencia en adultos de mediana edad; son ligeramente más frecuentes en mujeres. Están asociados con alteraciones de las sensaciones nerviosas que pueden ir desde la anestesia hasta la disestesia y el dolor manifiesto. Aunque el dolor se ha considerado un síntoma distintivo de esta lesión, los estudios indican que solo una cuarta parte a un tercio de los NTs orales son dolorosos ${ }^{1}$. Este dolor puede ser intermitente o constante y varía desde una leve sensibilidad o ardor hasta un dolor intenso que se irradia. Los neuromas del nervio mentoniano con frecuencia son dolorosos, especialmente cuando son palpados o se ven afectados por una dentadura postiza ${ }^{1}$.

Los NTs son lesiones poco frecuentes; en 2005, Jones y Franklin encontraron una frecuencia del $0,34 \%{ }^{5}$, Salla y cols. en 2009 encontraron una frecuencia del 0,07\% ${ }^{6}$. Además, en un estudio de 50000 muestras de biopsia de cavidad oral, solo se encontraron una frecuencia del $0,02 \%{ }^{7}$.

Los NTs son benignos, con frecuencia dolorosos agrandamientos de los nervios que aparecen después de un traumatismo o transección. Los neuromas del nervio infraorbitario se han informado previamente después de una cirugía de descompresión para el tratamiento de la enfermedad ocular tiroidea ${ }^{8}$.
Un NT es una lesión hiperplásica causada por un traumatismo o cirugía que involucra los nervios periféricos y no se considera una neoplasia verdadera. Las características clínicas de un NT incluyen la formación de un nódulo solitario de menos de $2 \mathrm{~cm}$ de diámetro, dolor neurálgico, sensibilidad, parestesia y aumento del dolor a la palpación sobre la lesión ${ }^{9,10}$.

Los NTs se originan en el extremo proximal de los nervios dañados y se encuentra en el tejido blando en lugar del hueso. Según un estudio de Peszkowski y Larsson ${ }^{11}$, que revisaron 48944 muestras de biopsia de la región oral, los NTs comprendieron solo el 0,09\% (45 casos). Aproximadamente un cuarto de esos neuromas eran lesiones intraóseas. Se considera poco probable que aparezca un NT en los huesos maxilares, porque la presión del hueso circundante restringe el crecimiento de la parte distal del nervio ${ }^{4,12}$.

En la región de cabeza y cuello, se ha informado que los neuromas ocurren con mayor frecuencia después de la parotidectomía y la disección del cuello, y menos después de la osteotomía mandibular y la extracción del diente ${ }^{13}$.

El origen de la reacción celular empieza cuando hay presencia de inflamación; la célula de origen empiezan a tornarse de forma esférica, su núcleo viaja a la periferia nerviosa al igual que los cuerpos de Nissl (acumulaciones basófilas del centro de la célula) que sufren un proceso llamado cromatolisis ${ }^{13}$. Este proceso se caracteriza por la inflamación del cuerpo neuronal; aumento del metabolismo celular; síntesis de proteínas, y una dispersión regional de los cuerpos de Nissl en la neurona, también hay una rápida aparición de conos de crecimiento en la punta proximal de los axones lesionados. A su vez el segmento distal del nervio sufre una "degeneración walleriana" que consiste en fragmentar al axón de su vaina de mielina tras la lesión para posteriormente conseguir la fagocitosis de los mismos ${ }^{14}$.

Se han recomendado métodos quirúrgicos y no quirúrgicos para el tratamiento de NTs sintomáticos. Se ha sugerido la infiltración local de esteroides, bloqueo simpático del ganglio, percusión y terapia ultrasónica. Las secciones del nervio y los bloqueos de alcohol no han tenido buenos resultados. También se han utilizado otras técnicas neuroquirúrgicas cuando las medidas locales no logran controlar el dolor. La resección quirúrgica simple de la lesión puede realizarse cuando el neuroma está bien localizado y se ha reportado que puede mejorar la sintomatología sin afectar el nervio ${ }^{9,10}$.

Ante la necesidad de diagnosticar oportunamente este tipo de lesiones de baja frecuencia y habiendo pocas publicaciones en nuestro medio que contribuyan a 
comprender su etiología, presentación clínica, diagnóstico y tratamiento consideramos la necesidad de reportar un caso clínico del manejo quirúrgico de un NT mandibular.

\section{Reporte del caso}

Se presenta una paciente de 23 años de edad que acude a la clínica de Cirugía Oral y Maxilofacial de la UNAM por presentar dolor y aumento de volumen en el fondo de surco vestibular mandibular del lado derecho a nivel de premolares.

Al interrogatorio directo la paciente menciona ser estudiante de odontología y que hace 1 año durante una práctica de anestesia recibió una punción directa sobre el nervio mentoniano lo que ocasionó dolor espontáneo de tipo ardoroso ${ }^{15}$ con una frecuencia de 2 a 3 veces al día con una evolución de aproximadamente cinco meses. Refiere que se utilizó anestesia local infiltrativa a base de mepivacaína al 2\% con epinefrina al 1:100 000 ppm y aguja dental corta (30 G).

Al examen clínico intraoral a nivel de los dientes $44 \mathrm{y}$ 45 se observa elevación de la mucosa alveolar, de color rosada, de consistencia firme, de aproximadamente 0,5 $\mathrm{cm}$ de diámetro (Figura 1) y dolor tipo ardoroso ${ }^{15}$ a la palpación. En la ortopantomografía no se observa ninguna alteración (Figura 2).

Debido a las características clínicas y la proximidad de la lesión con la emergencia del nervio mentoniano se sospe-

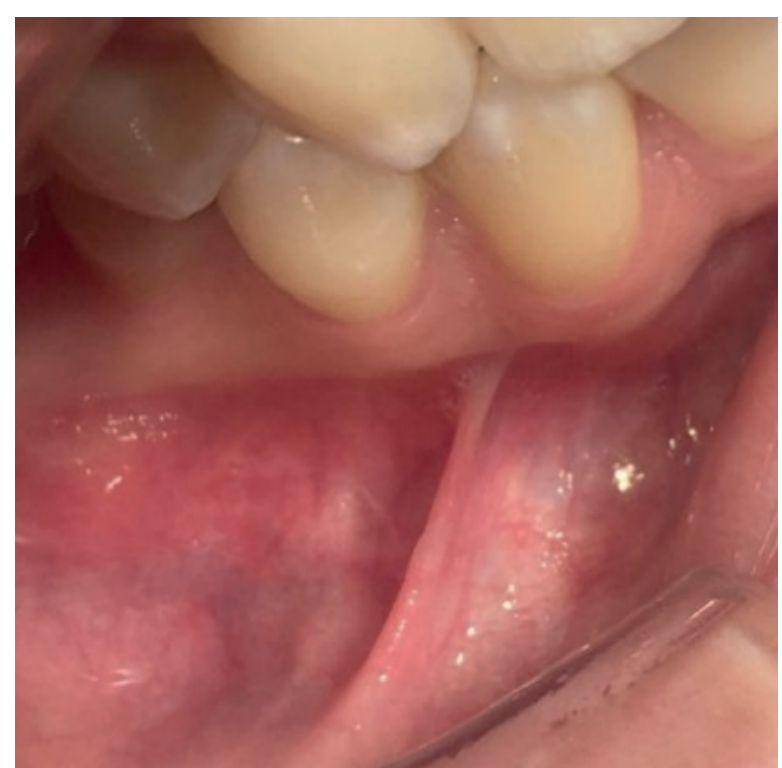

Figura 1. Foto intraoral de la lesión

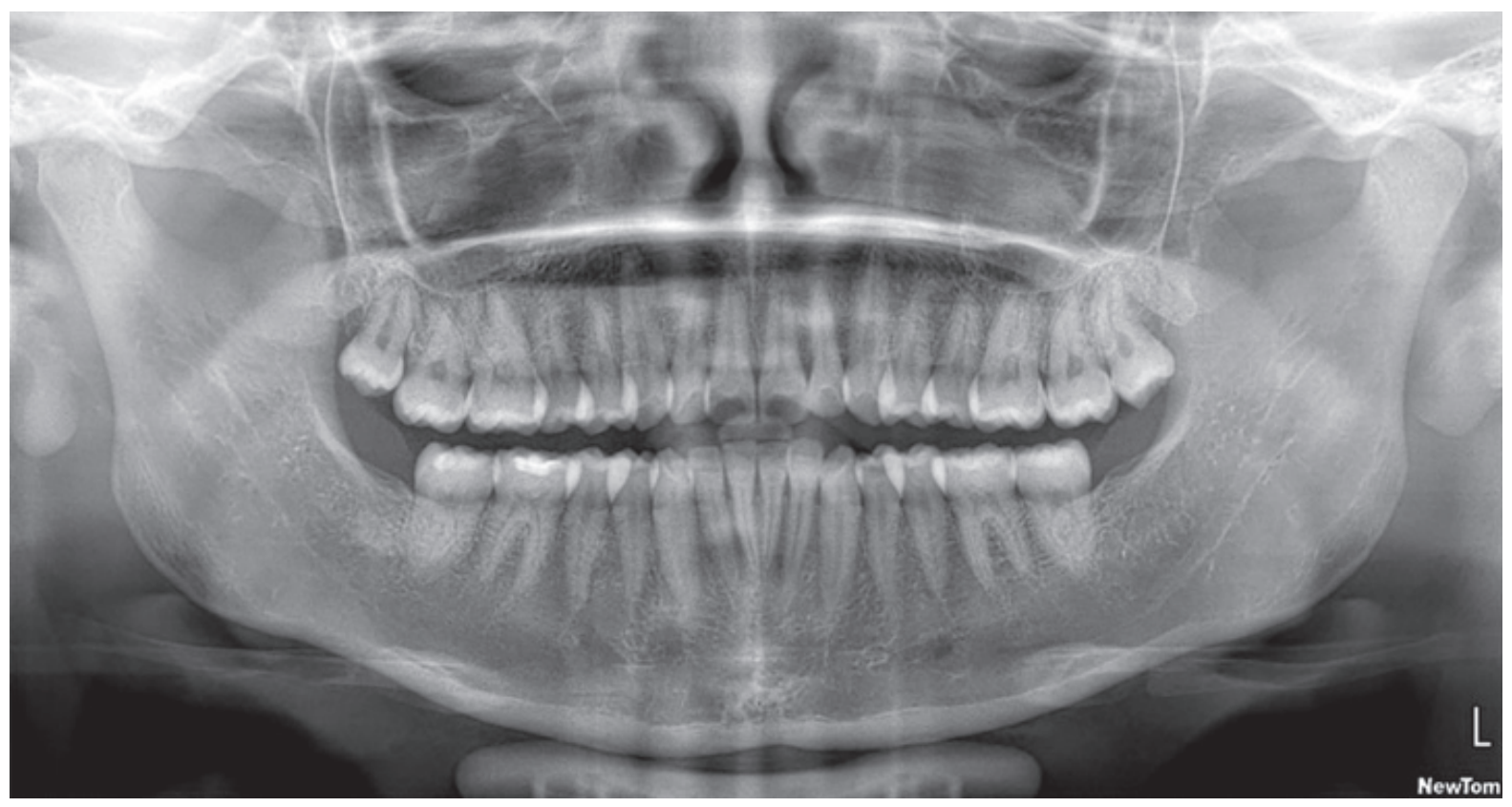

Figura 2. Ortopantomografía. No se observa evidencia de lesiones o alguna otra alteración ósea 
cha de una lesión neural, es así que se decide realizar una cirugía exploratoria con el diagnóstico presuntivo de NT.

Se procede a infiltrar con técnica de anestesia regional el nervio dentario inferior del lado derecho utilizando mepivacaína al 2\% con epinefrina al 1:100 000 ppm, posteriormente se diseña una incisión horizontal a nivel del fondo de surco vestibular entre los ápices de los dientes 44 y 45, seguida de una disección roma hasta evidenciar una lesión dependiente del nervio mentoniano que ocupaba todo el diámetro de la vaina nerviosa, de aproximadamente $0.5 \mathrm{~cm}$ por lo que se decide realizar una resección quirúrgica (Figura 3). Previamente se le explicó a la paciente los posibles tratamientos con ventajas y desventajas en caso de evidencia clínica de un NT, así como de las limitaciones de equipamiento de nuestra institución para un tratamiento más especializado, por lo que el tratamiento más conveniente en estas condiciones fue la escisión quirúrgica la cual fue consentida por la paciente.

Se obtiene un especímen de forma ahusada y de color blanco coral que involucra la rama nerviosa labial, gingival y mentoniana. En el lecho quirúrgico, se observa el agujero mentoniano después de la escisión libre de lesión macroscópica (Figura 4), por lo que finalmente se hace la síntesis de la herida utilizando una sutura simple continua con vicryl 4-0.

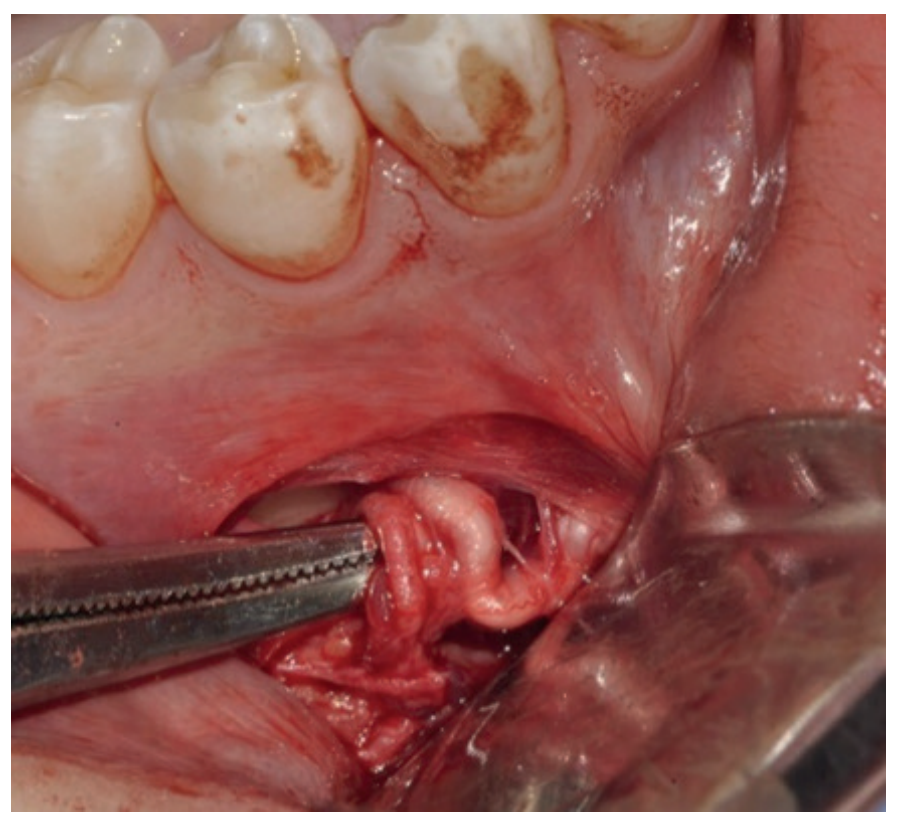

Figura 3. Lesión de tipo neural (nervio mentoniano)

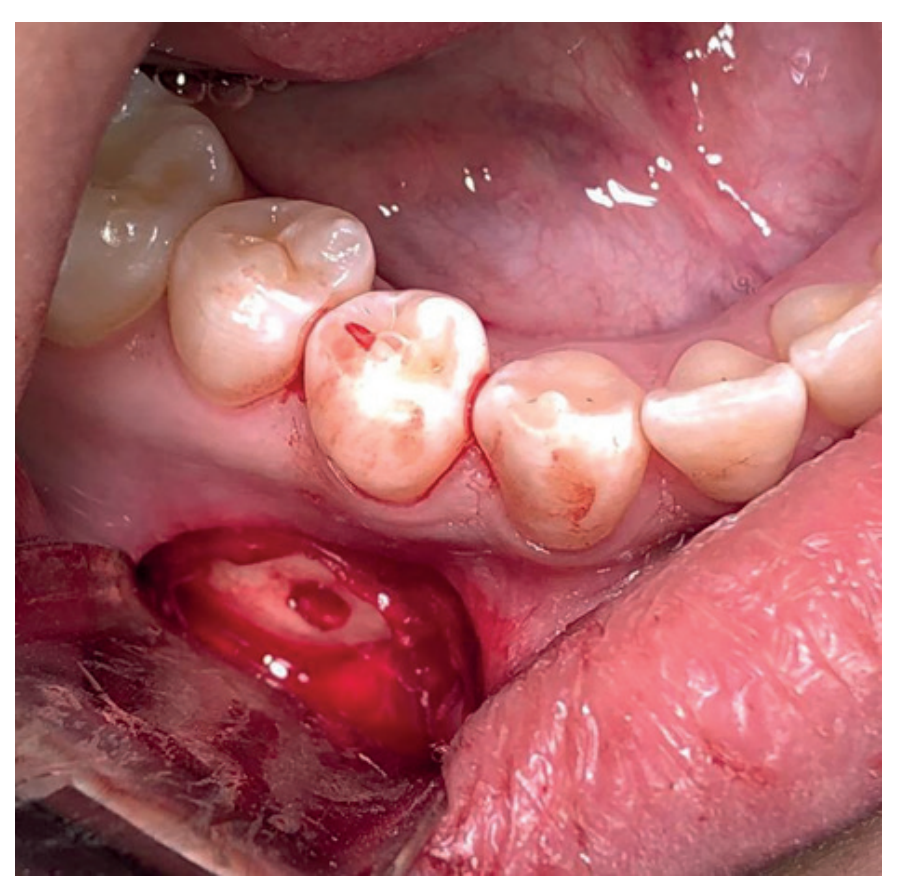

Figura 4. Lecho quirúrgico 
Se confecciona una mentonera de compresión para evitar el riesgo de formación de hematoma a nivel de las regiones bucal y submentoniana. Como parte del protocolo farmacológico postquirúrgico establecido en nuestro servicio se medica a la paciente con amoxicilina 500 mg una tableta cada ocho horas por siete días y paracetamol $500 \mathrm{mg}$ una tableta cada ocho horas por cinco días y se da cita para seguimiento clínico a los siete días.

El tejido extraído es colocado en formol al 10\% amortiguado y enviado para su estudio histopatológico al Departamento de Patología, Medicina Bucal y Maxilofacial de la División de Estudios de Posgrado e Investigación de la Facultad de Odontología de la Universidad Nacional Autónoma de México.

A los 7 días de seguimiento se observa edema en la región bucal y mentoniana del lado derecho así como anestesia a nivel de la piel del mentón y labio inferior del mismo lado. Se observa la herida quirúrgica con buena cicatrización sin evidencia de infección por lo que se decide retirar los puntos de sutura.

Macroscópicamente se describió como una muestra única de tejido blando que mide $2,3 \times 0,7 \times 0,4 \mathrm{~cm}$ de forma ahusada irregular, superficie irregular, color café claro con áreas beige y consistencia ahulada (Figura 5).

Para el análisis microscópico se utilizaron cuatro tinciones diferentes para su estudio diagnóstico: Hematoxilina - Eosina (H\&E), donde se observa proliferación de fibras pequeñas, basófilas y alargadas (neurofilamentos amielínicos), células de citoplasma claro con una fibra nerviosa dispuesta de manera central con el núcleo dispuesto en la periferia (neurofilamentos mielínicos en corte transversal) y que en algunas áreas forman vainas (longitudinal). Todo esto rodeado por tejido conjuntivo fibroso denso bien vascularizado, áreas de hemorragia reciente y escaso tejido adiposo (Figura 6A). Con la tinción Tricrómica de Masson, se observó en color rojo los haces nerviosos bien delimitados tanto de neurofilamentos amieilinicos como los mielinicos. De color azul débil se observa el tejido conectivo que compone al endoneuro, azul-grisáceo el epineuro y azul intenso la colágena (Figura 6D).

Por inmunohistoquímica se determinó la inmunorreactividad de pS100 y el antígeno epitelial de membrana (EMA). La proteína pS100 nos permitió observar como los fascículos contienen axones positivos para neurofilamentos tanto mielinicos como amielínicos y positividad en el citoplasma de las células de Schwan (Figura 6B). Con EMA, se observó positividad citoplasmática focal en células perineurales presentes en la pseudocápsula (perineuro) de la lesión (Figura 6C). El resultado histopatológico definitivo fue neuroma encapsulado traumático (de amputación).

En su segundo control a los 14 días no se evidencia clínicamente edema en la región bucal y mentoniana del lado derecho. A 10 meses de seguimiento se mantiene la anestesia de la región intervenida como consecuencia de la neurotmesis producida por la resección quirúrgica del nervio mentoniano. La paciente se ha mantenido asintomática y sin datos de recidiva.

\section{Discusión}

García y cols. en el 2008 mencionaron que la lesión se desarrolla con mayor frecuencia en los tejidos blandos del área del foramen mentoniano, el labio inferior y la

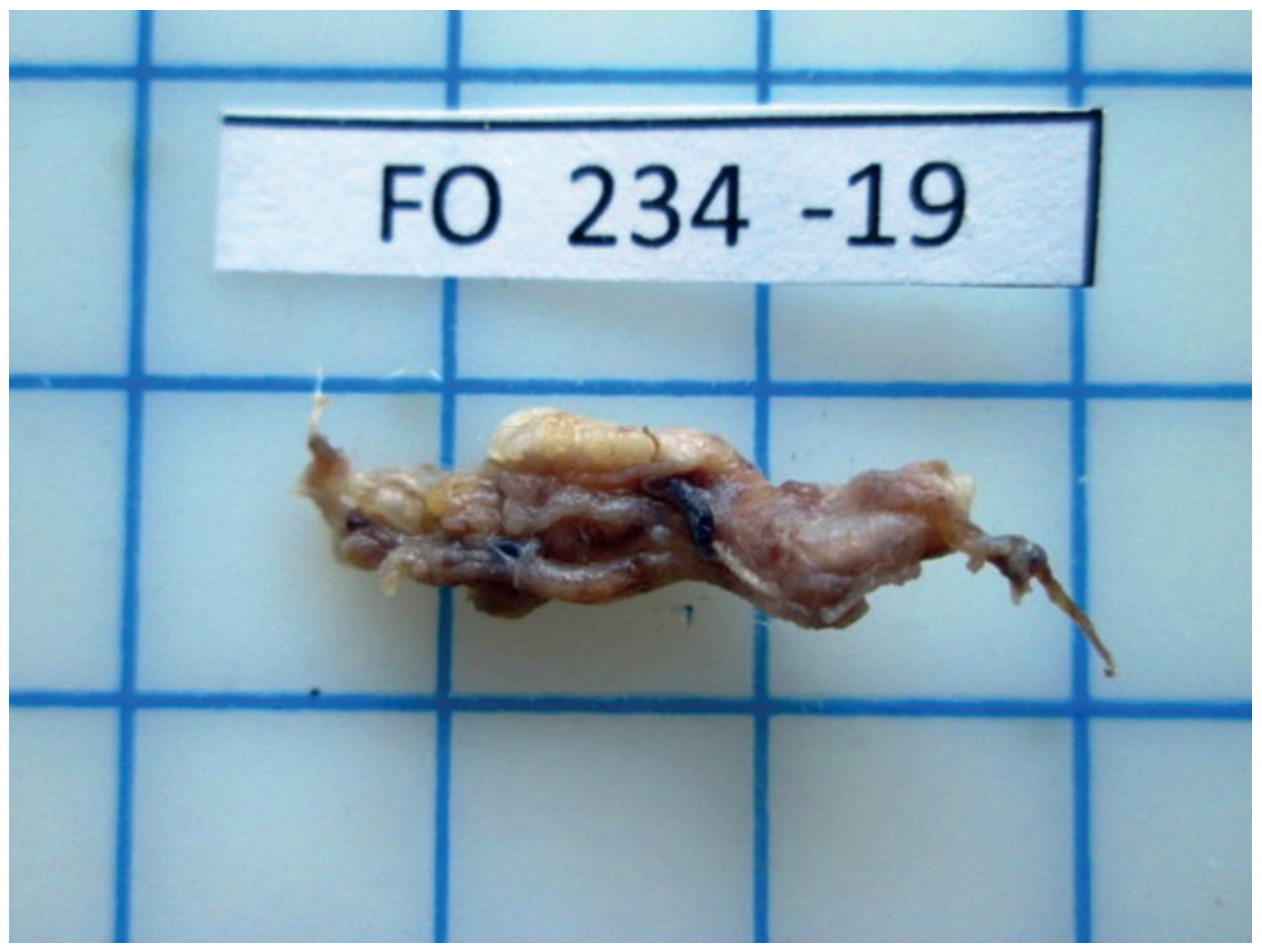

Figura 5. Pieza quirúrgica fijada en formol para su análisis macroscópico 


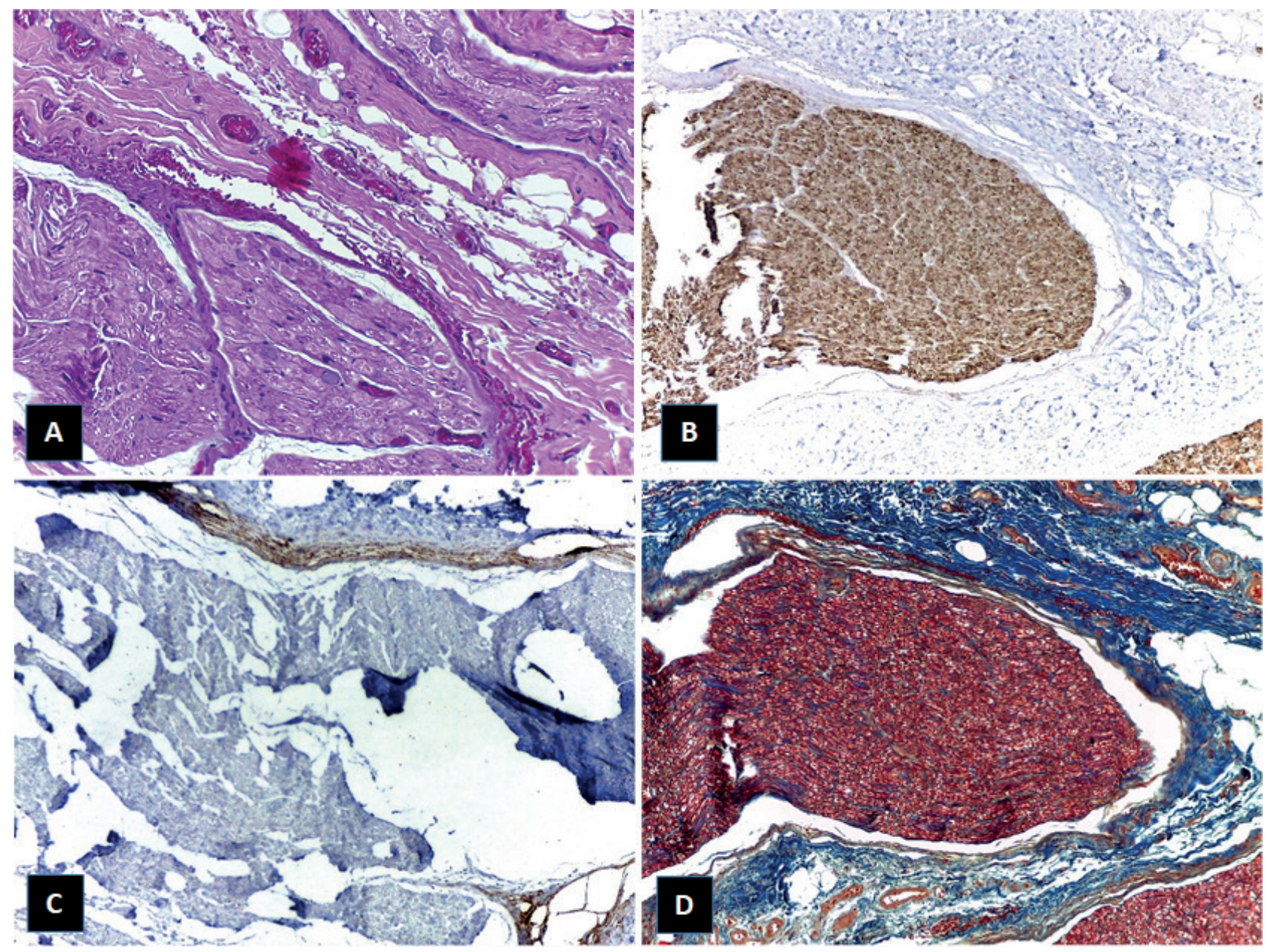

Figura 6. A. Corte histopatológico con tinción de H\&E a 400x. B. Corte histopatológico con tinción de pS100 a 10x. C. Corte histopatológico con tinción de EMA a 10x. D. Corte histopatológico con tinción de tricrómica de Masson a 10x.

lengua ${ }^{20}$. Al compararlo con nuestro caso se relaciona con su aparición a nivel de la emergencia del agujero mentoniano, afectando sus tres ramas.

Debemos tener presente en la historia clínica los antecedentes de trauma o cirugía que pudo haber tenido el paciente; ya que serán puntos clave para el diagnóstico. Interrogar sobre tratamientos dentales que hayan requerido anestesia local, puede orientarnos a la posible etiología como en el caso de nuestra paciente ${ }^{16}$. Debido a los signos clínicos y a la ubicación anatómica de la lesión, sólo se usó como ayuda diagnóstica la toma de una ortopantomografía para descartar que la lesión no fuera intraósea. En la mayoría de los casos cuando se tiene sospecha de la presencia de un NT se pueden utilizar varios auxiliares diagnósticos como ultrasonido, tomografía y resonancia magnética ${ }^{12,18}$.

Histológicamente en nuestro caso el NT se describió como una lesión hiperplásica del nervio identificándose múltiples haces nerviosos con axones, células de Schwann asociadas y una envoltura de tejido neural tal como lo describen Sapp y cols. y Stepic y cols ${ }^{17,19}$. Se dice que el NT va a estar conformado por cuatro variables: estroma, vascularización, infiltrado inflamatorio y tejido neural ${ }^{18}$. El estudio histopatológico en nuestro caso utilizando la tinción de EMA evidenció que el neuroma no es una verdadera neoplasia sino una proliferación de fibras nerviosas rodeada de un epineuro ya que sólo tiene reacción con esta capa, además la proteína S100 se usó para delimitar tejido neural afectado, y por último la tinción tricrómica de Masson evidenció la presencia de neurofilamentos mielínicos y amielínicos identificando cada una de sus capas $^{21}$.

Según Eguchi y cols. mencionan que existen NTs con límites difusos que impiden su resección quirúrgica respetando la vaina nerviosa; por lo que puede resultar en un daño neurológico severo, como un área de hipoestesia y parálisis nerviosa ${ }^{3}$, o como en nuestro caso anestesia debido a la imposibilidad anatómica de ser resecado de forma simple y conservadora como lo refieren Sayan y cols. y Chau y cols. en sus estudios ${ }^{9,10}$. Por lo tanto la resección quirúrgica en estos casos sería el tratamiento más indicado ${ }^{22}$.

Otras opciones terapéuticas de segunda línea citadas por diferentes autores son la radiocirugía estereotáctica, la infiltración local de esteroides, el bloqueo simpático del ganglio, la percusión y la terapia ultrasónica. Las secciones nerviosas o el bloqueo con alcohol 
han demostrado ser ineficaces e incluso pueden ser perjudiciales ${ }^{23}$.

Según Biglioli y cols. lo mejor es reconstruir a través de microcirugía un nervio dañado de inmediato. Los primeros signos de lesión nerviosa a menudo ocurren el día después de la cirugía, por esta razón, es necesario esperar un tiempo para evitar una intervención quirúrgica innecesaria, pero la neurorrafia generalmente se recomienda en caso de hipoestesia persistente 6 meses después de la lesión. En nuestro caso no optamos por la neurorrafia debido a la falta de equipos especializados para realizarla en nuestro servicio ${ }^{24}$.

El caso clínico presentado en este reporte demostró que la resección quirúrgica del NT resultó en una resolución completa del dolor; teniendo en cuenta que ha pasado casi un ańo sin recurrencia de los síntomas y que la paciente reportó mejoría desde la primera semana posterior a la cirugía. También observamos que se produjo una disestesia a nivel de la piel del labio inferior y mentón del lado derecho, la cual se confundía con una sensación de ardor durante las dos primeras semanas, para posteriormente terminar en una anestesia total de la zona.

\section{Referencias bibliográficas}

1. Neville B, Allen C, Damm D, Chi A. Oral and Maxillofacial Pathology; 4th edition. Canadá: Elsevier; 2016. Chapter 12, Soft Tissue Tumors; p. 489-90.

2. Rasmussen OC. Painful traumatic neuromas in the oral cavity. Oral Surg Oral Med Oral Pathol. 1980;49:191-5.

3. Eguchi T, Ishida R, Ara H, Hamada Y, Kanai I. A diffuse traumatic neuroma in the palate: a case report. J Med Case Rep. 2016;10:116.

4. Sist TC Jr, Greene GW. Traumatic neuroma of the oral cavity. Report of thirty-one new cases and review of the literature. Oral Surg Oral Med Oral Pathol. 1981;51(4):394-402.

5. Jones AV, Franklin CD. An analysis of oral and maxillofacial pathology found in adults over a 30-year period. J Oral Pathol Med. 2006;35:392-401.

6. Salla JT, Johann AC, Lana AM, do Carmo MA, Nunes FD, Mesquita RA. Immunohistochemical study of GLUT-1 in oral peripheral nerve sheath tumors. Oral Dis. 2008;14:510-3.

7. Peszkowski MJ, Larsson A. Extraosseous and intraosseous oral traumatic neuromas and their association with tooth extraction. J Oral Maxillofac Surg. 1990;48:963-7.

8. Amjadi S, Onn W, Rajak S, Morrisey D, Simon S, Davis G, Selva D \& Psaltis A. A case of traumatic infraorbital neuroma. Oral Maxillofac Surg. 2017; 21(4):471-473.
9. Sayan NB, Uçok C. Asymptomatic traumatic neuroma after mandibular sagittal split osteotomy: a case report. J Oral Maxillofac Surg. 2002;60(1):111-112.

10. Chau MN, Jönsson E, Lee KM. Traumatic neuroma following sagittal mandibular osteotomy. Int J Oral Maxillofac Surg. 1989;18(2):95区98.

11. Peszkowski MJ, Larsson A. Extraosseous and intraosseous oral traumatic neuromas and their association with tooth extraction. J Oral Maxillofac Surg. 1990;48(9):963-7.

12. Ngamsom S, Nakamura S, Kabasawa Y, Harada H, Tohyama R, Kurubayashi T. Imaging findings of intraosseous traumatic neuroma of the mandible. Oral Radiol. 2018;34(3):257-261.

13. Swanson HH. Traumatic neuromas. Oral Surgery Oral Medicine Oral Pathology. 1961;14:317-26.

14. Patodia S, Raivich G. Role of transcription factors in peripheral nerve regeneration. Front Mol Neurosci. 2012;5:8.

15. Orr PM, Shank BC, Black AC. The Role of Pain Classification Systems in Pain Management. Crit Care Nurs Clin North Am. 2017;29(4):407凶418.

16. Kiernan J. Barr. El sistema nervioso humano: Una perspectiva anatómica. 9a Edición. España: Editorial LWW; 2014. Capítulo 2, Células del Sistema Nervioso Central; p. 29.

17. Sapp J, Eversole L, Wysocki G. Contemporary oral and maxillofacial pathology. $2^{\text {nd }}$ Edition. St. Louis, Mo.: Mosby; 2004. Capítulo 9, Lesiones del Tejido Conjuntivo; p. 292-93.

18. Sist, TC, Greene GW. Traumatic neuroma of the oral cavity. Oral Surgery Oral Medicine Oral Pathology. 1981;51(4):394-402.

19. Stepic J, Pejovic M, Dragovic M et al. Traumatic neuroma of mental nerve following lower lip mucocele excision. Serbian Dental Journal. 2016;63(2):91-95.

20. Jham BC, Costa NL, Batista AC, Mendonça EF. Traumatic neuroma of the mandible: A case report with spontaneous remission. J Clin Exp Dent. 2014;6(3):317-20.

21. Fuertes L, Santonja C, Kutzner H, Requena L. Inmunohistoquímica en dermatopatología: revisión de los anticuerpos utilizados con mayor frecuencia (Parte I). Actas Dermo-Sifiliográficas. 2013;104(2):99-127.

22. Devassy J, Nayar BR. Biopsy- A Crucial diagnostic aid for oral lesions. IOSR-JDMS. 2018;17(9):16-24.

23. Arribas-García I, Alcalá-Galiano A, Gutiérrez R, Montalvo-Moreno JJ. Traumatic neuroma of the inferior alveolar nerve: a case report. Med Oral Patol Oral Cir Bucal. 2008;13(3):186-8.

24. Biglioli F, Lozza A, Colletti G, Allevi F. Objective Assessment of Lingual Nerve Microsurgical Reconstruction. J Craniofac Surg. 2018;29(8):e740冈e744. 
